\title{
Design and Implementation of Remote Medical Monitoring System for
}

\author{
Homecare \\ Juan Zhong ${ }^{1, a}$, * Hua Liao ${ }^{2, b}$ \\ ${ }^{1}$ College of Information Engineering, Gannan Medical University, Ganzhou, Jiangxi, China, 341000 \\ 2 STATE GRID GANZHOU ELECTRIC POWER SUPPLY COMPANY, Ganzhou, Jiangxi, China, \\ 341000 \\ aemail, ${ }^{b}$ email \\ *Corresponding Author: Juan Zhong
}

Keywords: Remote Homecare Monitoring System, Hardware Design, Software Design, Java

\begin{abstract}
With the development of computer and communication technology, the rapid development of telemedicine technology has been realized. Patients can communicate with the doctors in the hospital in time via the remote medical monitoring system. Based on the background, this paper presents the design and implementation of a set of remote homecare monitoring system, which includes the hardware system and the software system. The system overcomes the limitations of traditional medical devices and improves real time performance and flexibility of medical monitoring system.
\end{abstract}

\section{Introduction}

With the gradual improvement of people's life quality, all kinds of needs are constantly improving, especially the medical service requirements. The traditional, single medical treatment has been unable to meet the needs of people's healthcare. Therefore, there is an urgent need for a new medical system and device. The rapid development of computer, network technology and the emergence of the sensor provide an opportunity for the development of telemedicine. The research of telemedicine system has far-reaching significance in medical treatment. It alleviates the problem of uneven distribution of medical personnel and medical devices. It can reduce the medical burden of the family, and give the patients more free time and space. The main goal of this paper is to design and implement a stable mobile remote medical monitoring system development framework of mobile remote medical monitoring system business solutions, and through the research summed up a set of applicable in the international hospital of general mobile telemedicine monitoring protection. This kind of system not only needs good reusability and stability, but also needs to be configurable and efficient.

The development language of the remote mobile medical monitoring system is the Java language. The language is very popular with developers in the process of software development. Java language is easier to understand in the use of the process. In terms of security, Java security level is also higher, so many companies and personnel are selected for the system to study the technology. Java's storage allocation model is one of the main ways to defend against malicious code. Java does not have pointers, so programmers can't get hidden inside and fake pointers to point to memory. More importantly, the Java compiler does not deal with storage arrangements, so the programmer cannot see the actual storage arrangements for the class by viewing the statement. The stored 
reference in the compiled Java code determines the actual storage address at the running time by the Java interpreter.

\section{Overall System Design}

Based on the characteristics and development trend of the existing remote family medical monitoring system, this paper presents a new remote family health care system. It has the functions of real-time acquisition, storage and display of the physiological parameters. It can also transmit the signals to the doctors via Ethernet remote monitoring terminal in the form of remote video call. Remote monitoring terminal first data storage and backup for the user's physiological parameters of the control, and then sent to a cooperative relationship of the community hospital. Community hospital doctors will receive the physiological signal data for analysis and diagnosis and give the corresponding recommendations, and then the results of the diagnosis and recommendations to the remote monitoring terminal. According to the requirements of system design, remote home health care system should have the following functions: a variety of RS232 serial communication for receiving the physiological data collected by the sensors and send commands to control the corresponding sensor; the remote communication function, which can be made through the Ethernet monitoring terminal and monitoring center for data transmission for remote communication; a good man-machine interface and user-friendly operation which display system comes with a detailed understanding of the situation of their own health; data analysis and processing ability, which can identify the data collected in a timely alarm when abnormal. It should have a good software interface, which can make medical staff do much more intuitive analysis of the data.

The structure of the remote medical homecare system is shown in Figure 1.

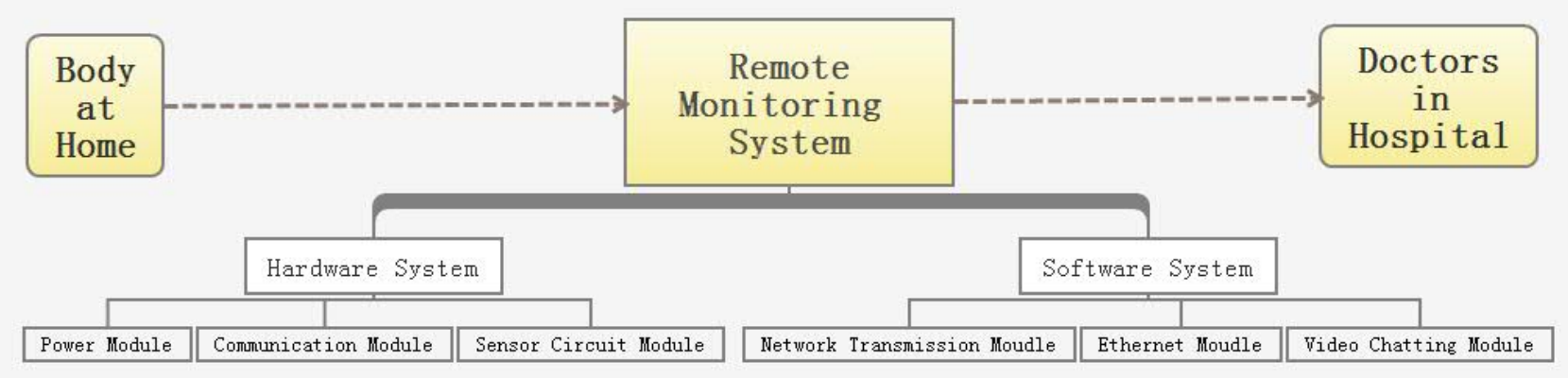

Figure 1.Structure of Remote Monitoring System

\section{Design and Implementation of Hardware System}

Power Module. The function of the power module is to provide energy sources for the entire system. Whether it can provide the corresponding voltage with the stability feature for the entire system has a great impact on the stable operation of the whole system. Especially for the acquisition of some sensitive physiological parameters, the noise interference is also an important influence factor. As the voltage required by each module is different, we need to provide a different module to match the voltage to ensure that it can work properly. We provide 3.3V voltage for the system through the LM1117 chip. LED liquid crystal display can generate images in the plane through the control of semiconductor light-emitting diodes. It has the characteristics of low energy consumption, low radiation, no flicker and reduces visual fatiSgue. This design uses a 2.4 inch TFT color touch screen to realize the functions of the ECG, blood pressure and pulse value display.

Communication Module. Through the standard Ethernet interface and broadband network connection, the network communication module is to realize the data transmission between the 
home and the monitoring center, the physiological parameters in real-time upload to the monitoring center. The doctors receive and analyze the data for the diagnosis in monitoring center and then give a timely feedback to the medical monitoring terminal. Traditional embedded system using the $10 \mathrm{MB} / \mathrm{s}$ Ethernet card has been unable to meet people's needs. This paper adopts has high transmission speed and high price of the chip as the main control chip of Ethernet module DS18B20. It do not need any external components, temperature range, high precision measurement, conversion speed, wear and impact resistance, small volume, convenient use, encapsulated in the form of diversity. DS18B20 is suitable for the digital temperature measurement and control field of all kinds of small space equipment. HK-2000G can be generated is proportional to the input pressure level of MV voltage output signal. It is driven by a voltage or current with excellent reproducibility and time stability. The product is not only small and very suitable for pulse rate detection, but also has a high sensitivity, strong anti-interference ability, large pressure range and so on.

Sensor Circuit Module. The sensor is the nervous system of the whole system in this design. It perceives various physiological parameters of the human body, and it is introduced into the main control chip to complete the collection of the parameters. ECG is the human heart in the periodic electrical activity, the body surface generated by the integrated bio electrical signal. ECG is the measurement of the surface of the human body generated by the cardiac activity and the potential energy generated by the graphics. By observing the electrocardiogram, it is helpful to diagnose many diseases, such as myocardial injury, myocardial infarction and coronary heart disease. Body temperature is not only a reflection of the body's metabolism, but also a necessary condition for the normal operation of the physiological activities of the human body. The normal body temperature can promote the body metabolism and life activities, and when the abnormal activities of life, the body's metabolism will corresponding malfunction of temperature change, so many of the symptoms of the human body can reflected by the temperature. According to the different contact methods, the body temperature measuring equipment can be divided into non-contact type temperature measuring instrument and contact type temperature measuring instrument. Contact type temperature measurement generally adopts the mercury thermometer measurement or thermistor measurement. Mercury thermometer is also widely used, but it contains mercury which is likely to cause mercury poisoning. The measurement is the most typical use of the human body's thermal effect, using pyro electric sensor to measure body temperature.

\section{Design and Implementation of Software System}

Network Transmission Module. The remote home health care terminal needs to connect with the network transmission module to realize the communication between the monitoring center and the hospital. Therefore, we need to establish a network transmission module. First of all, the system monitors the network card and configures the network card after the relevant parameters. If the network card is not monitored, the driver can initialize the parameters of the network card. When the STM32 chip transmits the data, first call the driver to send the program, and then write the data into the storage space, through the configuration of the activation of physical transmission, and finally through the interrupt to interrupt processing program. If at the time of the transmission, the data is wrong, the network card first produces the interrupt. The processor calls the interrupt processing procedure, carries on the corresponding processing. The Android mobile phone monitoring software needs to achieve three different monitoring functions. Through the WIFI access, the mobile phone software directly receives the transmission of vital signs data, real-time display of the phone is the guardian of the temperature value, attitude and pulse waveform. Through 
the web page, the project server PC receives information and stores it in the database. The mobile phone software is responsible for access as a server, public address to access the dynamic web page, and remote access is the guardian of the body temperature, attitude, heart rate and pulse rate. When you need to check the vital signs of the guardian, you can receive and send messages to the transmission side and display the physical sign information effectively.

Ethernet Module. The specific idea of the Ethernet module design is the network card detection for the operating system. When the card is found, the test process automatically configures the parameters of the network card. If you cannot find the parameters of the network card, the driver before running on the parameters of the network card set to complete the initialization of the network card. When the S3C2440 sends data, it can call the driver program to send a program. The data to be written into the storage space can enable physical transmitting process through the configuration. If the network card receives the data transmission process to be wrong, the network card will automatically produce the interrupt. The processor will call the interrupt processing procedure. At the same time, it carries on the judgment of the cause of the interruption and carries out corresponding treatment.

Video Chatting Module. When a video request is made by a user, a video request is first sent to the remote monitoring center, and the staff of the monitoring center can arrange the time according to the total reservation request. If the user makes a request for an appointment, the doctor can make a video call by selecting the appropriate user. At the same time, in the personal information table shows the current user's information and video call interface. It is convenient for the doctor in the video call with the user also can be more convenient understanding to the user's current physiological information and, if doctors on the user's current information uncertainty can also through the historical information and information management module and the physiological parameters in contrast. This further ensures the correctness of the diagnostic results. The terminal monitoring end of the remote home health care system is saved with the network port configuration of the current machine. Therefore, the configuration is required to write the relevant information to the memory. The data would be saved even if the power is lost. When the device starts, the system will take the relevant parameters automatically.

\section{Conclusion}

Adopting the advanced embedded technology, this paper designs a homecare remote medical monitoring system with the features of small size, multi-function, intelligence and humanism. The medical monitor terminal is connected with the hospital through Ethernet. The doctors can understand the health of the users in time to realize the function of remote monitoring. Due to the time pressure, there are many imperfect aspects needing continues research to make the homecare monitoring system more intelligent.

\section{References}

[1] Wang Zhongsheng, Huang Dongli, Chen Guoshao, Yang Sen, Modern Electronics Technique, Vol. 37 (2014) No 2, p.98-100

[2] Dai Yinhua, Li Hongmu, Zhao Weiyi, He Jianfeng, Electronics World, Vol. 34 (2012) No 7, p.42-43 
[3] S Santhi, GS Sadasivam,, Design and development of compact monitoring system for disaster remote health centers, Indian Journal of Medical Microbiology, Vol. 54 (2015) No 3, p.16-22

[4] Shi Daosheng, Ren Yi, Luo Huiqian, Journal of Wuhan University of Technology, Vol. 30 (2008) No 3, p.394-397 